Article

\title{
Tunable Polarization Gratings Based on Nematic Liquid Crystal Mixtures Photoaligned with Azo Polymer-Coated Substrates
}

\author{
Mateusz Nieborek ${ }^{1}$, Katarzyna Rutkowska ${ }^{1}$ (D), Tomasz Ryszard Woliński ${ }^{1}$, \\ Bartosz Bartosewicz $^{2}$ (D), Bartłomiej Jankiewicz ${ }^{2}$ (D) Dariusz Szmigiel ${ }^{3}$ and \\ Anna Kozanecka-Szmigiel ${ }^{1, *}$ \\ 1 Faculty of Physics, Warsaw University of Technology, 75 Koszykowa Str., 00662 Warszawa, Poland; \\ mateusz.nieborek.stud@pw.edu.pl (M.N.); Katarzyna.Rutkowska@pw.edu.pl (K.R.); \\ tomasz.wolinski@pw.edu.pl (T.R.W.) \\ 2 Institute of Optoelectronics, Military University of Technology, Kaliskiego 2, 00908 Warszawa, Poland; \\ bartosz.bartosewicz@wat.edu.pl (B.B.); bartlomiej.jankiewicz@wat.edu.pl (B.J.) \\ 3 Sieć Badawcza Łukasiewicz-Instytut Technologii Elektronowej, Al. Lotnikow 32/46, 02668 Warszawa, \\ Poland; szmigiel@ite.waw.pl \\ * Correspondence: anna.szmigiel@pw.edu.pl
}

Received: 9 August 2020; Accepted: 28 August 2020; Published: 31 August 2020

\begin{abstract}
Liquid crystal polarization gratings are of great interest for optical communications as elements performing beam steering, splitting, multiplexing or beam combining. Material birefringence, cell thickness or a period of the liquid crystal director pattern influence, among other features, spectroscopic and electro-optical characteristics of fabricated devices, determining thus their functionality and applicability. Here, we report on liquid crystal polarization gratings that allow for complete maximization of the first-order diffraction efficiency (resulting in total elimination of the zeroth-order diffraction) for any wavelength of an incident beam from green to the near-infrared spectral region by applying a low electric voltage. The gratings with periods as small as $10 \mu \mathrm{m}$ were obtained by holographic exposure of the cell substrates coated with light-sensitive azo polymer alignment layers, and then filled with three different liquid crystal mixtures. The influence of gold nanoparticle dopants in the liquid crystalline mixtures on spectroscopic and electro-optical properties of the devices is presented. Moreover, on the basis of the measured transmittance spectra of the fabricated gratings, the unknown birefringence of liquid crystal mixtures as well as their effective birefringence due to molecular reorientation in the electric field in the visible and near IR region were determined.
\end{abstract}

Keywords: photoalignment; diffraction grating; polarization grating; effective birefringence

\section{Introduction}

Diffraction gratings are optical elements with periodic structures spatially modulating the amplitude or/and phase of an incident light. Importantly, the efficiency of output beam generation strongly depends on the grating type. Less typical among conventional gratings are polarization gratings inducing periodic changes in the polarization state of the output optical field [1]. Polarization gratings may be inscribed in suitable photoresponsive materials by exposing them to light interference patterns formed by two coherent waves of mutually orthogonal polarizations [2,3]. As a result of such an irradiation, periodic changes in the induced optical birefringence (i.e., anisotropy of the refractive index) or/and in a direction of the induced optical axis of the medium are generated. Unique diffraction properties have been predicted for the gratings recorded with the equal-intensity beams that possess 
opposite circular polarization and cross at small angles (superposition of such waves results in an optical field of a constant intensity and linear polarization state with polarization azimuths that continuously change along the interference region) [3-5]. Such a configuration guarantees that, firstly, the generated diffraction orders are not higher than the first ones. Secondly, the \pm 1 -order diffracted beams may appear without the zeroth-order (i.e., non-diffracted) beam if the induced birefringence $\Delta n$ and material thickness $d$ satisfy the so-called half-wave retardation condition at a given probing wavelength $\lambda: \Delta n d=(m-1 / 2) \lambda, m$-integer number. Finally, the first-order diffraction efficiencies are highly sensitive to the polarization state of the probing beam. At a half-wave retardation condition, it is theoretically possible to direct a linearly polarized incident beam completely into both of the first diffraction orders or to direct a circularly polarized incident beam completely into a single (either +1 or -1) diffraction order depending on the polarization handedness. Thus, it is possible to reach up to $50 \%$ or $100 \%$ first-order diffraction efficiency, respectively.

Azobenzene-containing polymers are well-known photoresponsive materials showing photoinduced optical anisotropy (i.e., photoinduced birefringence or/and dichroism) upon irradiation with linearly polarized light of a proper wavelength [6-9]. Selective light absorption processes by rod-like azo molecules and multiple trans-cis-trans isomerizations of azo molecules lead to their reorientation into directions perpendicular to the polarization direction, which gives rise to optical anisotropy. Numerous experiments have shown that irradiation of azo polymer layers with two beams of opposite circular polarizations results in efficient diffraction of the probe beam by volume birefringence (polarization) grating and surface relief grating (SRG) almost simultaneously formed in the material [10-13]. The latter, being sinusoidal variations of a sample thickness, additionally modulates the phase of the incident wave, increasing the intensity of the first-order diffraction signal. According to Viswanathan et al. [14], efficiency of SRG formation is strongly sensitive to a recording geometry. The authors showed that the process is significantly suppressed when using the contra-circularly polarized beams that cross at small angles (below $5^{\circ}$ ), which is the recording geometry required for inscription of pure polarization gratings described by L. Nikolova and T. Todorov [3]. Considering typical values of light-induced birefringence in azo polymers and typical thickness of polymeric layers (ca. $0.015 \div 0.05$ and $1 \mu \mathrm{m}$, respectively), it can be roughly estimated that volume birefringence gratings generated in these materials introduce phase retardations much below the half-wave retardation condition. As a consequence, the generated first-order diffracted beams are of low intensities.

Efficiency of the polarization gratings created in azo polymers may be strongly enhanced by utilization of liquid crystals (LCs). Due to anisotropic intermolecular interactions, liquid crystal molecules may align according to azo molecule orientation pattern when placed between the azo polymer-coated substrates of an LC cell. Implementation of LCs has another advantage over passive diffraction gratings formed in azo polymer layers. First, there is a large variety of available compounds and mixtures whose wide range of accessible material birefringence values can be additionally easily adjusted not only by temperature but also by adding specific dopants. Moreover, implementation of LCs allows for fabrication of tunable elements due to the sensitivity of liquid crystal molecules to electric fields and a resulting possibility of a precise control of the effective birefringence values by an applied voltage. The LC-based polarization gratings offer diverse and important applications in photonics, e.g., in projection displays, beam steering, multiplexing, beam splitting or combining devices [15-19]. Recently, interesting approaches for the realization of LC-based tunable polarization gratings were reported [20,21].

Despite the well-recognized ability of azo polymers to uniformly align liquid crystals [22-24], the vast majority of photoaligned LC gratings have been demonstrated using other groups of light-sensitive materials, such as photocrosslinkable polymers, pure azo dyes or azo-dye-doped polymers [16,25-28]. Functionalized azo polymers (with covalently attached azo moieties) constitute, however, a wide and diverse group of materials, whose physico-chemical properties, optical response or anchoring strength to align liquid crystals may be tailored by an almost unlimited number of structural 
modifications. Unlike doped azo polymers, the functionalized systems offer significantly increased thermal stability of the induced azo molecule arrangement after irradiation, which is extremely important from the point of view of practical applications. Moreover, reversible photochemistry in azo polymers allows for recording of erasable and rewritable LC alignment patterns, which is not possible with photocrosslinkable polymers. Very recently, we have reported on a successful fabrication of tunable LC polarization gratings based on azo poly (amide imide) layers irradiated with two beams of opposite circular polarizations [29]. Regrettably, a period of the obtained LC structure was ca. $23 \mu \mathrm{m}$, what resulted in an unsatisfactory separation between diffracted and non-diffracted beams.

Here, we report our progress in the realization of tunable small-period LC polarization gratings based on azo polymer alignment surfaces. We focus our attention on the influence of gold nanoparticle concentration in the nematic LC mixtures on diffraction and electro-optical properties of the obtained gratings. Moreover, using a white-light beam, we monitor the changes in the grating transmittance with applied voltage. From the measured transmittance spectra at $0 \mathrm{~V}$ and at higher voltages, we calculate the unknown values of LC mixture material birefringence as well as effective birefringence, practically in the whole visible/near-IR spectral range.

\section{Experimental}

\subsection{Materials}

The chemical structure of the functionalized azo polymer (PAI) used as photoaligning material as well as its synthesis route and physicochemical properties have been reported in [30]. The polymer possesses two azobenzene moieties per a repeating unit covalently attached to the poly (amide imide) backbone via one of the phenyl rings of the azo moiety.

The 4-cyano-4'-pentylbiphenyl (5CB) nematic LC was modified with $3 \%$ of $4^{\prime \prime}$-n-pentyl-4-cyanop-terphenyl. Subsequently, the modified LC (termed as LC_0) was doped with gold nanoparticles (AuNPs) using the method reported elsewhere [31]. Gold nanoparticles were synthesized in chloroform following the Brust-Schiffrin method, which allows one to obtain highly stable, dodecanethiol functionalized NPs of the sizes of $1 \div 3 \mathrm{~nm}$ [32]. The LC-AuNPs composites with various AuNP content were fabricated by mixing colloidal suspension of AuNPs in chloroform with LC_0 and slow evaporation of the solvent. Two composites (LC_1, LC_2) with different concentrations of AuNPs were prepared, namely with 0.1 and $0.5 \%$ wt. of nanoparticles, respectively.

\subsection{Preparation of the LC Diffraction Gratings}

The N-methyl-2-pyrrolidone (NMP) solution of the PAI azo polymer (1 wt.\%) was filtered through $0.45 \mathrm{~mm}$ membranes and spin-coated at $2500 \mathrm{rpm}$ on indium tin oxide-coated glasses in a clean-room environment. Subsequently, the plates were dried at $130{ }^{\circ} \mathrm{C}$ for $1.5 \mathrm{~h}$ in a vacuum oven. The thickness of the PAI layers was ca. $30 \mathrm{~nm}$ as measured with a Dektak XT stylus profiler.

Three LC cells with the measured gap $(d)$ in the range $4.6 / 4.8 \mu \mathrm{m}$ were assembled from the PAI-coated substrates using $5 \mu \mathrm{m}$ glass spacers. Then, the empty cells were exposed to a light interference pattern formed by two coherent $442 \mathrm{~nm} 40 \mathrm{~mW} / \mathrm{cm}^{2}$ beams (from a He-Cd laser) with orthogonal circular polarizations (a detailed scheme of the experimental set-up was presented in our recent paper [29]). In the arranged geometry, the beams crossed at the angle $(\theta)$ of ca. $2.5^{\circ}$. This led to a resulting optical field characterized by a constant intensity and linear polarization with a direction that rotates with a period $(\Lambda)$ of ca. $10 \mu \mathrm{m}$ along the interference area (according to a formula: $\Lambda=\lambda /(2 \sin (\theta / 2))$ [14]. After $80 \mathrm{~min}$ irradiation, the cells were filled with three different LC mixtures (LC_0, LC_1, LC_2) using the capillary action at room temperature. 


\subsection{Measurements of Spectroscopic and Electro-Optical Properties of the LC Gratings}

The grating transmittance spectra in the range of $370 / 900 \mathrm{~nm}$ were measured as a function of applied voltage by detecting an intensity of zeroth-order diffracted waves using an incident white-light beam and a portable UV-vis spectrometer (USB 2000 Ocean Optics).

Electro-optical switching characteristics of the two LC gratings (i.e., LC_0 and LC_1) were studied using linearly polarized $690 \mathrm{~nm}$ probe beam in the experimental set-up presented in Figure 1. The intensity of the +1 st-order diffracted beam as a function of time was monitored by a silicon photodetector and an oscilloscope (Tektronix DPO4104). The LC cells were driven with $1 \mathrm{kHz}$ AC signal (from a function generator Rigol DG4062 working in an ASK mode) with an amplitude being changed between ca. $1.3 \mathrm{~V}$ and ca. $2.1 \mathrm{~V}(f=1 \mathrm{~Hz})$ to determine the time periods, in which the transmitted $690 \mathrm{~nm}$ beam was redirected from the 0th to \pm 1 st diffraction orders, and vice versa. The measured rise and fall times were defined as the time intervals, in which the intensity of the +1 st diffraction order changed from $10 \%$ to $90 \%$ and from $90 \%$ to $10 \%$ of the maximum value, respectively.

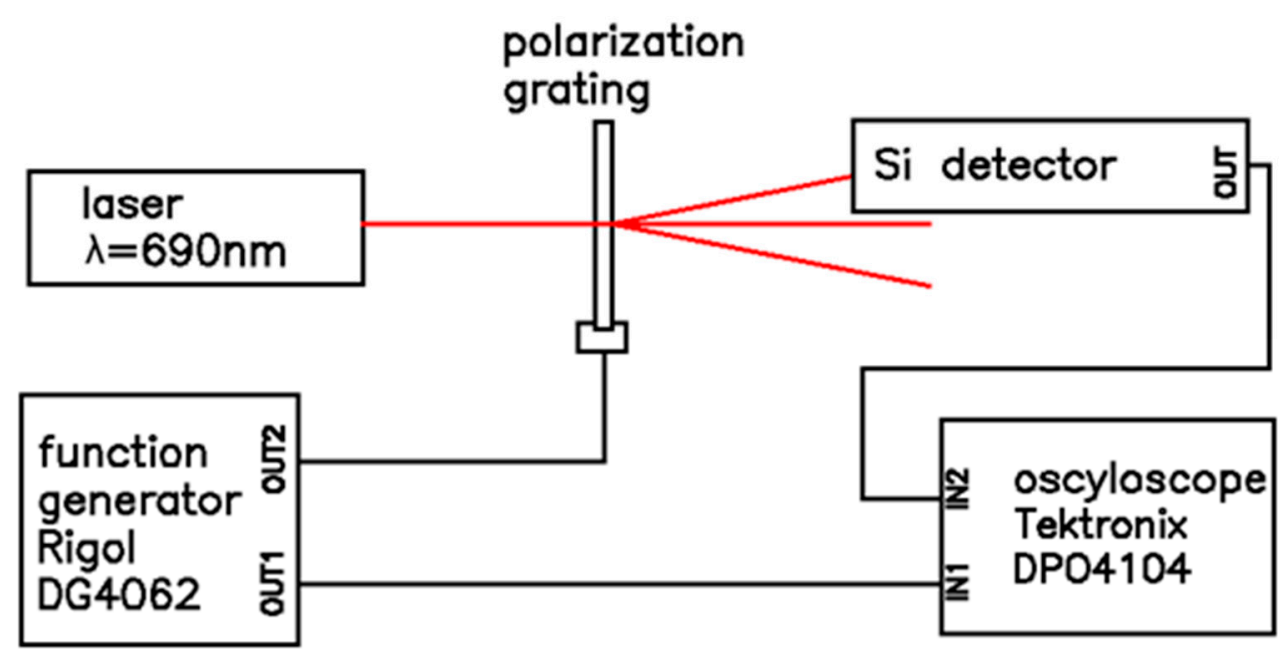

Figure 1. Experimental set-up for measurements of switching times of the prepared liquid crystal (LC) gratings.

\section{Results and Discussion}

Figure 2a,d show an image of the LC_1 photopatterned cell under a polarizing microscope between crossed polarizers at $0 \mathrm{~V}$ and a schematic drawing of the expected LC director orientation in the bulk of the cell, respectively.

A regular pattern demonstrating good-quality LC alignment was clearly visible. The spacing between the neighboring dark areas read from the photo was $5.5 \mu \mathrm{m}$, that is, half the period of the arranged interference pattern $(\Lambda / 2)$, which indicated that the LC director distribution in the cell followed a designed azo molecule orientation in the PAI substrates. Indeed, for the LC cell with molecules aligned as presented in Figure $2 \mathrm{~d}$, the regions with a director orientation along a polarizer or analyzer axis appear dark under crossed polarizers. Similar images were also found for the prepared LC_0 and LC_2 cells.

Diffraction patterns created by all fabricated LC cells were tested using a normally incident $690 \mathrm{~nm}$ probe beam of a linear polarization. Specifically, the changes in the generated patterns observed with an applied voltage for the LC_1 cell are exemplarily presented in Figure 3; the corresponding optical microscope images of the cell between the crossed polarizers are shown in Figure 2b,c. 

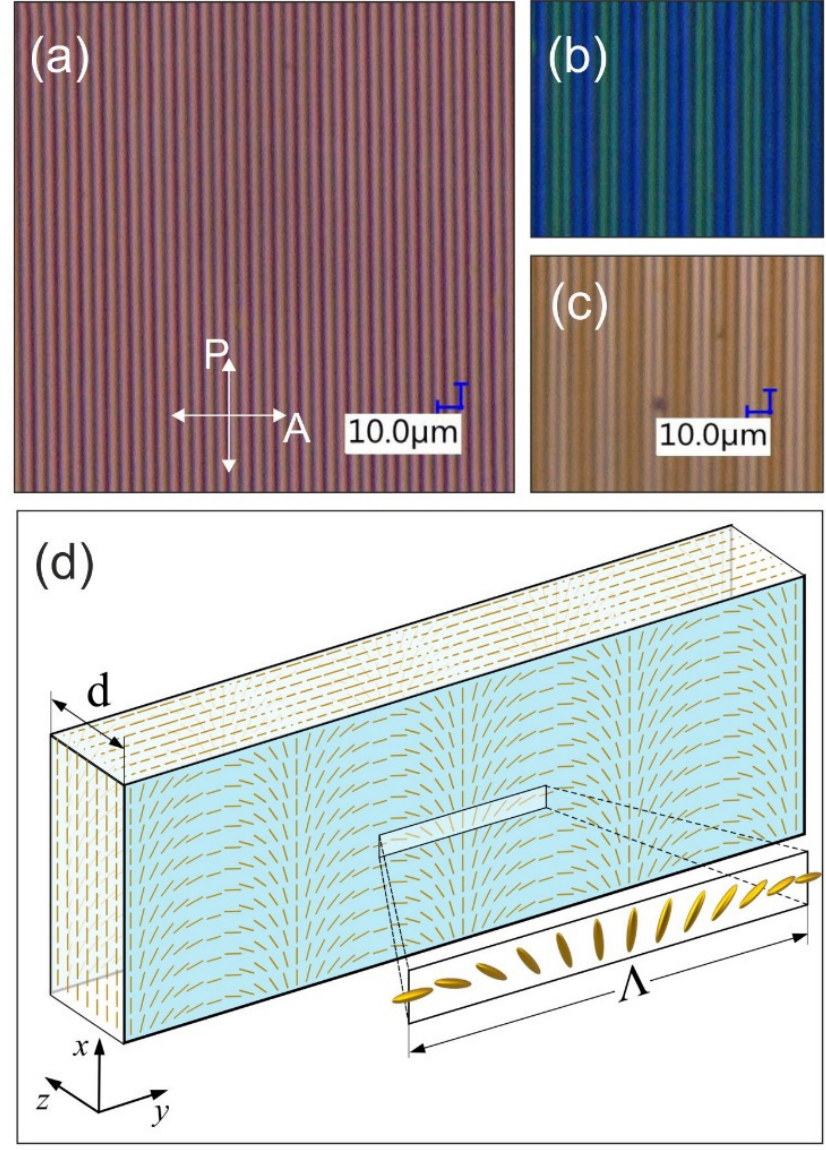

Figure 2. Optical microscope image of the LC_1 cell placed between the crossed polarizers (a) at $0 \mathrm{~V}$, (b) at $1.35 \mathrm{~V}$, (c) at $2.2 \mathrm{~V}$; (d) schematic drawing of the expected LC director orientation in the bulk of the cell at $0 \mathrm{~V}$.
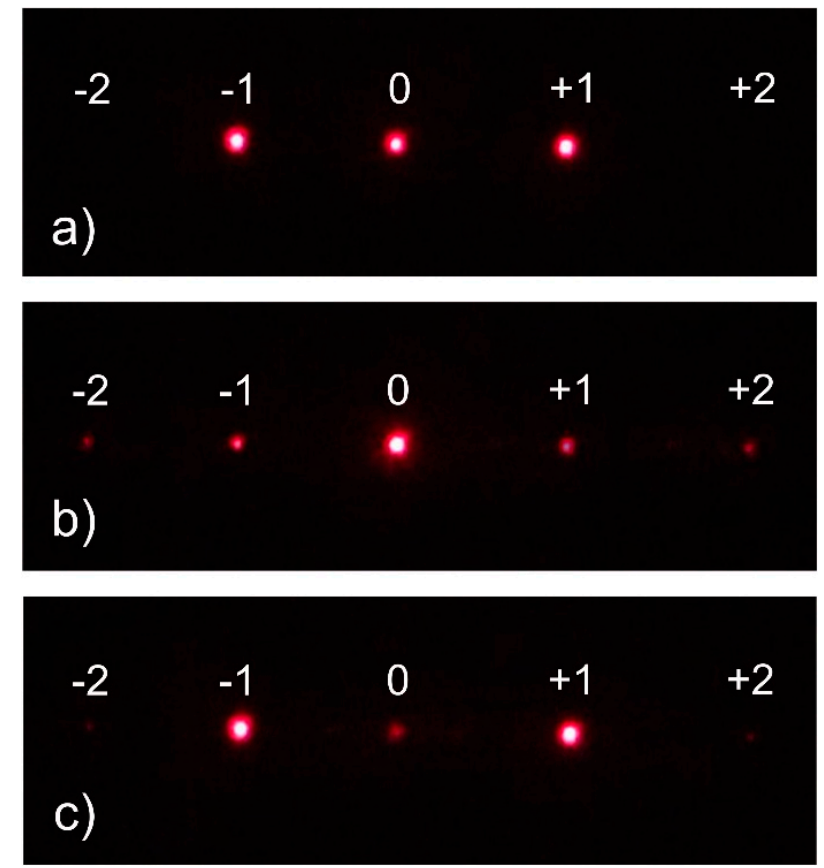

Figure 3. Diffraction patterns observed for the linearly polarized $690 \mathrm{~nm}$ beam incident onto LC_1 cell at (a) $0 \mathrm{~V}$, (b) $1.35 \mathrm{~V},(\mathbf{c}) 2.2 \mathrm{~V}$. 
At zero voltage, three beam spots, i.e., the central 0 order and both +1 st- and -1 st-order spots were clearly seen (Figure 3a). From simultaneous presence of the three beams behind the grating, it was evident that the birefringence of the LC_1 mixture at $690 \mathrm{~nm}$ and the cell gap were not adjusted to the half-wave retardation condition. However, by applying the electric voltage across the cell (of a value exceeding a threshold voltage of Freedericksz transition), the effective birefringence of LC_1 might be continuously varied, leading to the effect of a dynamic interchange between intensities of the diffracted beams. Thus, at $1.35 \mathrm{~V}$, the intensity of the non-diffracted beam was maximized, while that of the +1 st and -1 st diffraction orders was minimized (Figure $3 b$ ). The half-wave retardation condition was then fulfilled at $2.2 \mathrm{~V}$, as demonstrated by the highest recorded intensity of both first-order beams and a diminished non-diffracted wave (Figure 3c). As shown in Figure $2 b, c$, the applied voltages changed the effective birefringence, while very regular patterns with unchanged spacing were seen, which confirmed the existing grating structures. Similar voltage-dependent diffraction patterns and similar values of steering voltages of $1.3 \mathrm{~V}$ and $2.1 \mathrm{~V}$ were found for the cell filled with the LC_0 mixture. However, in the case of the LC_2 cell, the undesired second- and third-order diffraction spots were clearly observed. The presence of additional diffracted beams behind the LC_2 grating indicated deviations in the LC director alignment pattern from that presented in Figure 2b, introduced by gold nanoparticles. Nevertheless, the above results confirmed the ability of irradiated PAI layers to align nematic LC mixtures periodically, with a period twice as dense as that presented recently [29]. It is important to note that successful fabrication of the gratings with a $10 \mu \mathrm{m}$ period allowed for increasing the diffraction angle of $690 \mathrm{~nm}$ light to $4^{\circ}$ compared to only $1.7^{\circ}$ for the mentioned LC polarization grating with the $23 \mu \mathrm{m}$ period [29]. Larger separation between the zeroth and the first orders increases an application potential of the fabricated optical elements and encourages future research efforts on further minimizing the LC structure period.

Spectroscopic properties of the prepared gratings were investigated by monitoring the 0thorder-transmittance using normally incident white-light beam. Figure 4 compares the un-normalized LC cell transmittance spectra in the absence of the applied voltage. The spectra were recorded with a reference transmission intensity taken in the absence of the LC cells.

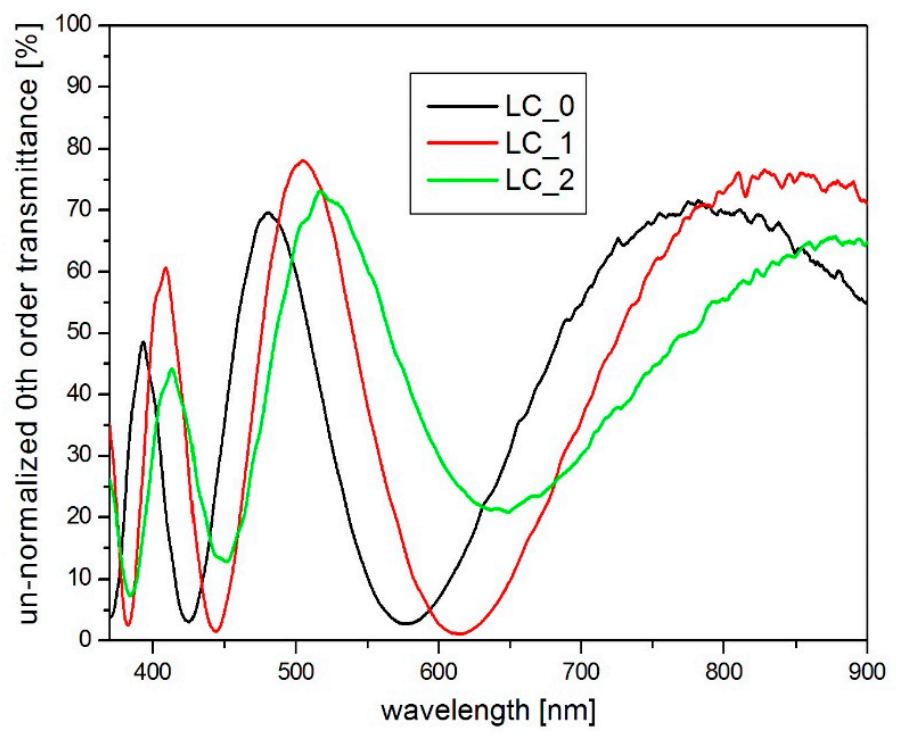

Figure 4. Un-normalized transmittance of the prepared LC gratings at zero voltage (recorded with a reference transmission intensity taken in the absence of the LC cells).

Note that the maxima of the spectra correspond to the wavelengths that are directed into the zeroth order alone, while the minima stand for the wavelengths that are completely redirected from the zeroth order. It is worth noting that the highest transmittances reached $70-80 \%$ due to scattering and reflection losses. Lower values of the maximum transmittances observed in the ultraviolet spectral range can 
be additionally attributed to absorption in azo polymer aligning layers as well as in ITO (Indium Tin Oxide) coatings. It can be seen from Figure 4 that at 0 V the LC_0 cell directed $480 \mathrm{~nm}$ or $785 \mathrm{~nm}$ light in the zeroth diffraction order alone, while $425 \mathrm{~nm}$ or $580 \mathrm{~nm}$ light was almost completely redirected from the zeroth order, resulting from the full- and half-wave retardation conditions, respectively. In case of the LC_2 cell, the zeroth-order intensity did not vanish completely for any wavelength, confirming unsuccessful formation of a pure polarization grating due to too large concentration of the nanoparticle dopants deviating the LC director profile from the desired one. Moreover, it can be noticed that the entire transmittance spectra of LC_ 1 and LC_2 were red-shifted relative to the spectrum of the LC_0 cell. As each cell had the same thickness, the shift indicated the differences in birefringence of the prepared mixtures.

Subsequently, the zeroth-order transmission spectra of the two obtained polarization gratings (i.e., LC_0 and LC_1) were examined at different external voltage values. In these measurements, the reference transmission intensities were recorded in such a way that a white-light beam illuminated the LC cell at the area non-exposed to light interference pattern before infiltration with LC. Therefore, the effects of reflection and absorption losses were eliminated (we termed these spectra as normalized ones). It was found that the threshold voltage needed for electrical tuning was very low and practically the same for LC_0 and LC_1 cells, i.e., equal to $1 \mathrm{~V}$. Above this threshold, the zeroth-order transmittance spectra were shifting continuously towards shorter wavelengths as a result of decreasing effective birefringence, as shown in Figure 5a, which illustrates the spectra recorded for the LC_ 1 grating at $0 \mathrm{~V}$, as well as at two steering voltages, i.e., $V_{1}=1.25 \mathrm{~V}$ and $V_{2}=2.2 \mathrm{~V}$. Such experimental observations are in perfect conformity with theoretical predictions presented in Figure $5 \mathrm{~b}$. The latter shows results of certain calculations and numerical simulations performed for the grating, illustrating influence of voltage-driven molecular reorientation on diffraction efficiency (and thus on the zeroth-order transmittance). Details of the performed numerical analysis with the specific form of the Euler-Lagrange differential equations, which were solved with the use of the relaxation scheme, are provided in our previous work [29]. Specifically, the inset in Figure $5 \mathrm{~b}$ represents numerically calculated spatial distribution of the molecular director within one period of the grating for the voltage above the threshold value. Such electrically induced changes in molecular arrangement with respect to that forced by the boundary conditions lead to the lowering of the effective birefringence with the voltage and, thus, to a blue shift and eventually to the reduction in the number of multiple spectral fringes over the analyzed spectral range.
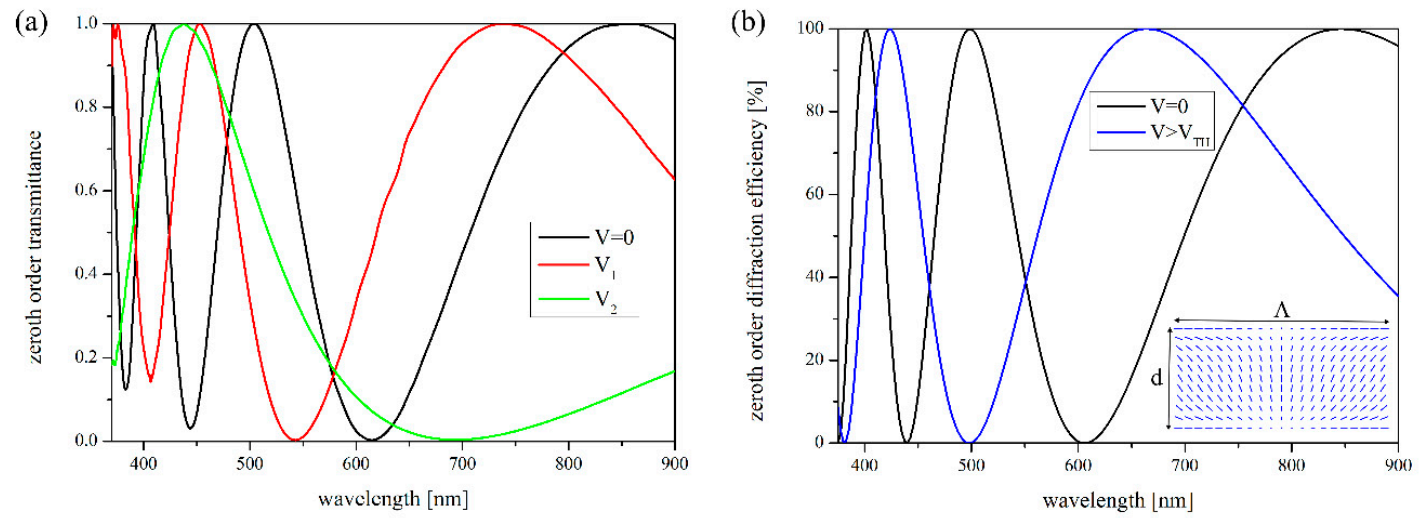

Figure 5. (a) Normalized zeroth-order transmittance spectra of the LC_1 grating at different voltages $\left(V_{1}=1.25 \mathrm{~V}\right.$ and $V_{2}=2.2 \mathrm{~V}$ ) showing the effect of grating tunability in experimental conditions. (b) Zeroth-order diffraction efficiency calculated numerically in the grating for no-voltage and at the voltage exceeding the Fredericks threshold $\left(\mathrm{V}_{\mathrm{TH}}\right)$ with $V_{1}<\mathrm{V}<V_{2}$. Inset illustrates voltage-induced changes in molecular orientation in the $y-z$ plane within one grating period. 
The obtained results prove that the phase difference between the ordinary and extraordinary waves is easily controlled by electric voltage so that the half-wave retardation condition may be satisfied for all wavelengths in the visible/near-IR region. A possibility of extinction of the non-diffracted beam of any wavelength demonstrates an achromaticity of the fabricated optical elements. Nevertheless, it should be pointed out that due to absorption of blue-violet wavelengths by azo polymer aligning layers, the gratings could be dedicated for use for higher probing wavelengths (i.e., above ca. $500 \mathrm{~nm}$ ).

The normalized transmittance spectra at $0 \mathrm{~V}$ allowed for calculating the unknown material birefringence $\left(\Delta n=n_{e}-n_{0}\right.$, where $n_{0}$ and $n_{e}$ are ordinary and extraordinary refractive indices, respectively) of the LC_0 and LC_1 mixtures as a function of wavelength in accordance with the zeroth-order diffraction efficiency defined in the general case as $\cos ^{2}\left(\pi d \Delta n_{\text {eff }} / \lambda\right)[4]$, where $\Delta n_{\text {eff }} \equiv \Delta n$ in the analyzed gratings at $\mathrm{V}=0$. Birefringence dispersion characteristics for these two mixtures are shown at the top part of the graph in Figure 6.

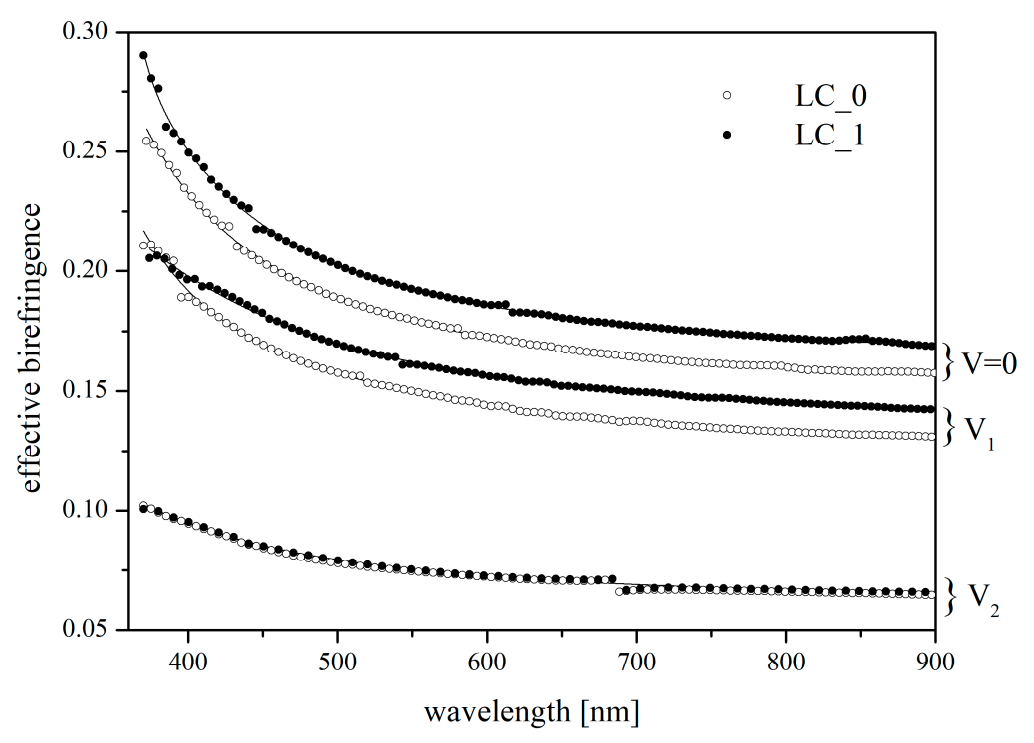

Figure 6. Birefringence $(\mathrm{V}=0)$ and effective birefringence (at $V_{1}=1.25 \mathrm{~V}$ and $V_{2}=2.2 \mathrm{~V}$ ) calculated for LC_0 and LC_1 mixtures based on the 0th-order diffraction efficiency registered in the entire spectral range. Discontinuities in the plots are related to periodicity of the cosine function.

As can be observed, the presence of the Au nanoparticle dopant resulted in an increase in the birefringence of the nematic LC mixture. Such correlations have been previously mentioned in the literature [33-35]. The grating transmittance spectra recorded at the chosen voltages $\left(V_{1}=1.25 \mathrm{~V}\right.$, $V_{2}=2.2 \mathrm{~V}$ ) allowed for determining the effective birefringence $\Delta n_{\mathrm{eff}}$, which is related to the LC director reorientation and is a function of the director orientation angles [29]. Please note that for clarity, the symbols in Figure 6 represents every twentieth measurement point (determined by the spectroscope resolution) and that the fitted functions following the three-parameters Cauchy model (solid lines) perfectly reproduce the shape of the theoretical curve given for pure 5CB LC [36].

Birefringence and its wavelength dependence are the fundamental parameters and characteristics of LCs. Their accurate measurements are very important for determining the retardation of LC-based optical devices, which is important for their design and operation. The applied method allowed for quick and simple characterization of the material characteristics in entire operation spectral range in a single measurement. This contrasts with other applied techniques in which birefringence is retrieved based on measurements performed for each wavelength independently, such as when using the Abbe refractometer [36], the wedge-cell [37], the Fabry-Perot etalon [38] or the Michelson [39] or Mach-Zehnder [40] interferometer. It also appears to be improved when compared to other spectroscopic methods in which the birefringence can be determined only at some particular 
wavelengths [41] or multiple measurements [42], S-transform [43] or advanced equipment [44] are required in order to obtain the birefringence of liquid crystal materials at any wavelength.

The switching behavior of the LC_0 and LC_1 cells were characterized by measuring the time periods required for redirecting a $690 \mathrm{~nm}$ probe beam between the zeroth and the first diffraction orders, which corresponded to switching a driving signal amplitude between $1.3 \mathrm{~V}$ and $2.1 \mathrm{~V}$ or between $1.35 \mathrm{~V}$ and $2.2 \mathrm{~V}$, for the LC_0 and LC_1 cell, respectively. The estimated rise time was $13.5 \mathrm{~ms}$ or $12 \mathrm{~ms}$ for the LC_0 and LC_1 cell, respectively, while the fall time was the same for both the cells and equals $44 \mathrm{~ms}$. Figure 7a,b show the exemplarily measured changes in the intensity of the first-order diffracted beam $(690 \mathrm{~nm})$ by the LC_1 cell.
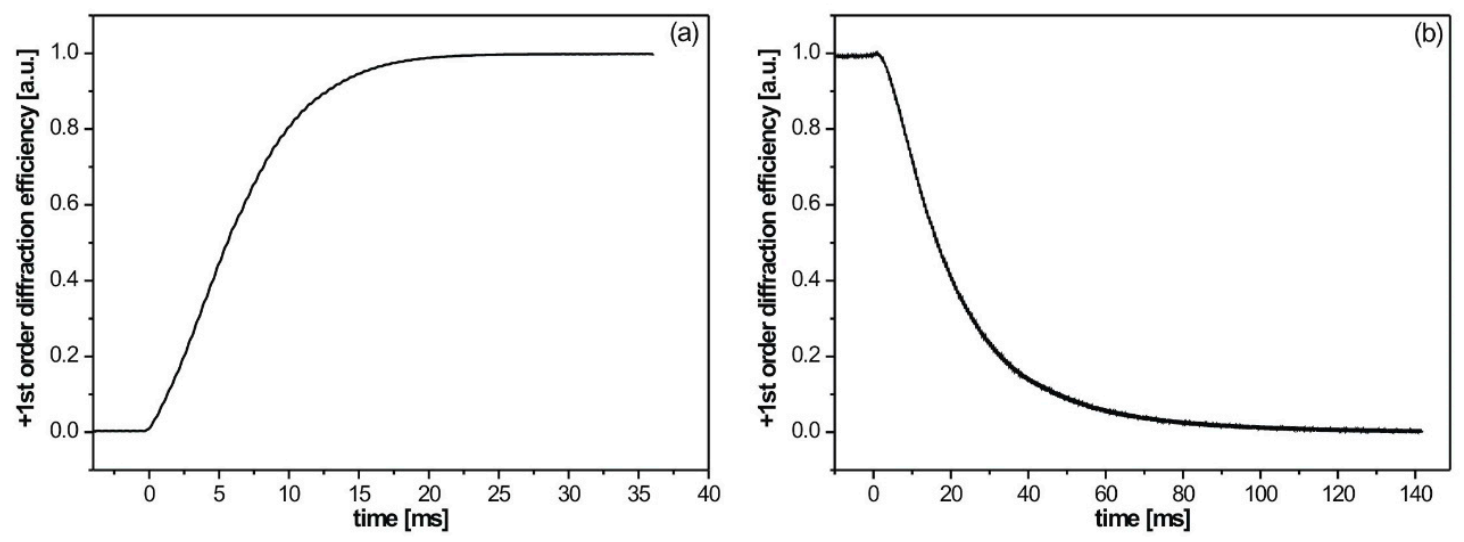

Figure 7. Intensity of the first-order diffracted $690 \mathrm{~nm}$ beam by the LC_1 cell as a function of time when changing a driving voltage amplitude from (a) $1.35 \mathrm{~V}$ to $2.2 \mathrm{~V}$, and (b) $2.2 \mathrm{~V}$ to $1.35 \mathrm{~V}$, which corresponded to switching the transmitted light between the zeroth and first diffraction orders.

It is important to underline that tuning voltages required for switching between the beams as well as the voltage difference are very low. For example, in the case of a red probe beam, the steering voltages differed by only $0.85 \mathrm{~V}$, and this can be considered as the main superiority of the proposed grating operation principle. The operation principle is not based on inducing a homeotropic alignment by external voltage (i.e., completely erasing the periodic structure of LC director) for maximization of the zeroth-order diffraction efficiency, as in the case of other LC gratings reported e.g., by Escuti and Jones [45] or by Weglowski et al. [19]. Therefore, lower steering voltages are used for operation of the LC gratings presented in this study.

\section{Conclusions}

Tunable and efficient diffraction gratings were fabricated using nematic liquid crystals, azo polymer-coated aligning substrates and polarization holography as a method for inducing the optical anisotropy in the polymer layers. The gratings were characterized by relatively small spacing of $10 \mu \mathrm{m}$, confirming the large applicational potential of the utilized functionalized azo polymer in the area of photoalignment of nematic liquid crystals.

Two of the three prepared gratings, i.e., these based on the undoped LC mixture (LC_0) and on the mixture doped with $0.1 \%$ wt. of gold nanoparticles (LC_1), belonged to the so-called polarization-type gratings generating only the zeroth and/or the first diffraction orders depending on the wavelength of an incident light. The third grating based on the LC mixture doped with $0.5 \%$ wt. of gold nanoparticles (LC_2) produced higher-order diffracted beams and, thus, belonged to classical phase-type gratings. It was shown that by applying a low electric voltage to the LC_0 and LC_1 cells, the intensities of the zeroth- and first-order diffracted beams could be continuously interchanged. In particular, changing the applied voltage between only ca. 1.3 and $2.2 \mathrm{~V}$ resulted in a complete redirecting of the passing $690 \mathrm{~nm}$ probe beam from the zeroth into the first diffraction orders and vice versa. The measured switching times were of order of $10 \mathrm{~ms}$. 
On the basis of measured transmittance spectra of the two obtained polarization gratings, the birefringence of utilized liquid crystal mixtures as well as their effective birefringence due to molecular reorientation in electric field were determined. The proposed single-measurement method appears accurate and fast, as it allows for determination of material birefringence in a wide spectral range.

Author Contributions: Conceptualization, T.R.W. and A.K.-S.; formal analysis, K.R. and A.K.-S.; funding acquisition, T.R.W.; investigation, M.N. and A.K.-S.; methodology, M.N., B.B., B.J. and D.S.; resources, B.B. and B.J.; visualization, K.R. and D.S.; writing—original draft, A.K.-S.; writing-review and editing, K.R. All authors have read and agreed to the published version of the manuscript.

Funding: This work was partially supported by the Polish National Science Centre under the grant no. UMO-2015/19/B/ST7/03650.

Acknowledgments: The authors thank Ewa Schab-Balcerzak and Jolanta Konieczkowska from the Centre of Polymer and Carbon Materials of the Polish Academy of Science for the PAI azo polymer synthesis, and Roman Dabrowski for fruitful discussion.

Conflicts of Interest: The authors declare no conflict of interest.

\section{References}

1. Cincotti, G. Polarization gratings: Design and applications. IEEE J. Quantum Electron. 2003, 39, 1645-1652. [CrossRef]

2. Kakichashvili, S.D. Hologram polarization recording. Optika Spektrosk. 1972, 33, 324-327.

3. Nikolova, L.; Todorov, T. Diffraction efficiency and selectivity of polarization holographic recording. Optica Acta 1984, 31, 579-588. [CrossRef]

4. Nikolova, L.; Ramanujam, P.S. Polarization Holography; Cambridge University Press: Cambridge, UK, 2009.

5. Xu, M.; de Boer, D.K.; van Heesch, C.M.; Wachters, A.J.; Urbach, H.P. Photoanisotropic polarization gratings beyond the small recording angle regime. Opt. Express 2010, 18, 6703-6721. [CrossRef] [PubMed]

6. Dumont, M.L.; Sekkat, Z. Dynamical study of photoinduced anisotropy and orientational relaxation of azo dyes in polymeric films: Poling at room temperature. Proc. SPIE 1993, 1774, 188-199.

7. Natansohn, A.; Rochon, P. Photoinduced motions in azo-containing polymers. Chem. Rev. 2002, 102, 4139-4176. [CrossRef] [PubMed]

8. Yager, K.G.; Barrett, C.J. Novel photo-switching using azobenzene functional materials. J. Photochem. Photobiol. A 2006, 182, 250-261. [CrossRef]

9. Hvilsted, S.; Sánchez, C.; Alcalá, R. The volume holographic optical storage potential in azobenzene containing polymers. J. Mat. Chem. 2009, 19, 6641-6648. [CrossRef]

10. Barrett, C.J.; Natansohn, A.L.; Rochon, P.L. Mechanism of optically inscribed high-efficiency diffraction gratings in azo polymer films. J. Phys. Chem. 1996, 100, 8836-8842. [CrossRef]

11. Lagugné Labarthet, F.; Buffeteau, T.; Sourisseau, C. Analyses of the diffraction efficiencies, birefringence, and surface relief gratings on azobenzene-containing polymer films. J. Phys. Chem. B 1998, 102, 2654-2662. [CrossRef]

12. Helgert, M.; Fleck, B.; Wenke, L.; Hvilsted, S.; Ramanujam, P. An improved method for separating the kinetics of anisotropic and topographic gratings in side-chain azobenzene polyesters. Appl. Phys. B 2000, 70, 803-807. [CrossRef]

13. Takase, H.; Natansohn, A.; Rochon, P. Photocrosslinked surface relief gratings on azobenzene-containing copolymer films. Polymer 2003, 44, 7345-7351. [CrossRef]

14. Viswanathan, N.K.; Balasubramanian, S.; Li, L.; Tripathy, S.K.; Kumar, J. A detailed investigation of the polarization-dependent surface-relief-grating formation process on azo polymer films. Jpn. J. Appl. Phys. 1999, 38, 5928. [CrossRef]

15. Sarkissian, H.; Park, B.; Tabirian, N.; Zeldovich, B. Periodically aligned liquid crystal: Potential application for projection displays. Mol. Cryst. Liq. Cryst. 2006, 451, 1-19. [CrossRef]

16. Oh, C.; Escuti, M.J. Achromatic diffraction from polarization gratings with high efficiency. Opt. Lett. 2008, 33, 2287-2289. [CrossRef]

17. Li, J.; Hu, X.; Wei, B.; Wu, Z.; Ge, S.; Ji, W.; Hu, W.; Lu, Y. Simulation and optimization of liquid crystal gratings with alternate twisted nematic and planar aligned regions. Appl. Opt. 2014, 53, E14-E18. [CrossRef] 
18. Park, J.; Yu, C.; Kim, J.; Chung, S.; Lee, S. Concept of a liquid-crystal polarization beamsplitter based on binary phase gratings. Appl. Phys. Lett. 2003, 83, 1918-1920. [CrossRef]

19. Weglowski, R.; Kozanecka-Szmigiel, A.; Piecek, W.; Konieczkowska, J.; Schab-Balcerzak, E. Electro-optically tunable diffraction grating with photoaligned liquid crystals. Opt. Commun. 2017, 400, 144-149. [CrossRef]

20. Chen, R.; Lee, Y.; Zhan, T.; Yin, K.; An, Z.; Wu, S. Multistimuli-Responsive Self-Organized Liquid Crystal Bragg Gratings. Adv. Opt. Mater. 2019, 7, 1900101. [CrossRef]

21. Xiong, J.; Chen, R.; Wu, S. Device simulation of liquid crystal polarization gratings. Opt. Express 2019, 27, 18102-18112. [CrossRef]

22. Lucht, S.; Neher, D.; Miteva, T.; Nelles, G.; Yasuda, A.; Hagen, R.; Kostromine, S. Photoaddressable polymers for liquid crystal alignment. Liq. Cryst. 2003, 30, 337-344. [CrossRef]

23. Yaroshchuk, O.; Reznikov, Y. Photoalignment of liquid crystals: Basics and current trends. J. Mat. Chem. 2012, 22, 286-300. [CrossRef]

24. Seki, T.; Nagano, S.; Hara, M. Versatility of photoalignment techniques: From nematics to a wide range of functional materials. Polymer 2013, 54, 6053-6072. [CrossRef]

25. Crawford, G.P.; Eakin, J.N.; Radcliffe, M.D.; Callan-Jones, A.; Pelcovits, R.A. Liquid-crystal diffraction gratings using polarization holography alignment techniques. J. Appl. Phys. 2005, 98, 123102. [CrossRef]

26. Kawai, K.; Sakamoto, M.; Noda, K.; Sasaki, T.; Kawatsuki, N.; Ono, H. Design and fabrication of a tunable wavelength-selective polarization grating. Appl. Opt. 2016, 55, 6269-6274. [CrossRef] [PubMed]

27. Presnyakov, V.; Asatryan, K.; Galstian, T.; Chigrinov, V. Optical polarization grating induced liquid crystal micro-structure using azo-dye command layer. Opt. Express 2006, 14, 10558-10564. [CrossRef] [PubMed]

28. Provenzano, C.; Pagliusi, P.; Cipparrone, G. Highly efficient liquid crystal based diffraction grating induced by polarization holograms at the aligning surfaces. Appl. Phys. Lett. 2006, 89, 121105. [CrossRef]

29. Kozanecka-Szmigiel, A.; Rutkowska, K.A.; Nieborek, M.; Kwasny, M.; Karpierz, M.A.; Schab-Balcerzak, E.; Konieczkowska, J.; Szmigiel, D. Photopatterned azo poly (amide imide) layers as aligning substrates of holographic liquid crystal diffraction gratings for beam steering applications. J. Mater. Chem. C 2020, 8 , 968-976. [CrossRef]

30. Konieczkowska, J.; Schab-Balcerzak, E.; Siwy, M.; Switkowski, K.; Kozanecka-Szmigiel, A. Large and highly stable photoinduced birefringence in poly (amideimide) s with two azochromophores per structural unit. Opt. Mater. 2015, 39, 199-206. [CrossRef]

31. Budaszewski, D.; Chychłowski, M.; Budaszewska, A.; Bartosewicz, B.; Jankiewicz, B.; Woliński, T.R. Enhanced efficiency of electric field tunability in photonic liquid crystal fibers doped with gold nanoparticles. Opt. Express 2019, 27, 14260-14269. [CrossRef]

32. Brust, M.; Walker, M.; Bethell, D.; Schiffrin, D.J.; Whyman, R. Synthesis of thiol-derivatised gold nanoparticles in a two-phase liquid-liquid system. J. Chem. Soc. Chem. Commun. 1994, 7, 801-802. [CrossRef]

33. Vardanyan, K.K.; Palazzo, E.D.; Walton, R.D. Nematic nanocomposites with enhanced optical birefringence. Liq. Cryst. 2011, 38, 709-715. [CrossRef]

34. Acreman, A.; Kaczmarek, M.; D'Alessandro, G. Gold nanoparticle liquid crystal composites as a tunable nonlinear medium. Phys. Rev. E 2014, 90, 012504. [CrossRef]

35. Tejaswi, M.; Rao, M.; Datta Prasad, P. Synthesis and characterization of citrate capped gold nanoparticles and their effect on liquid crystals: Optical studies. J. Chem. 2016, 9, 697-705.

36. Li, J.; Wen, C.; Gauza, S.; Lu, R.; Wu, S. Refractive indices of liquid crystals for display applications. J. Disp. Technol. 2005, 1, 51. [CrossRef]

37. Rutkowska, K.; Kozak, A.; Orzechowski, K. Chromatic dispersion measurements of selected liquid crystalline materials for integrated optics applications. Proc. SPIE 2016, 10034, 100340J.

38. Ko, M.O.; Kim, S.; Kim, J.; Lee, B.W.; Jeon, M.Y. Measurement of effective refractive index of nematic liquid crystal in Fabry-Perot etalon. J. Opt. Soc. Korea 2015, 19, 346-350. [CrossRef]

39. Abbas, B.; Khalil, M.A. An Experimental Method for Determination of the Refractive Index of Liquid Samples Using Michelson Interferometer. Acta Phys. Pol. A 2016, 129, 59. [CrossRef]

40. Inam, M.; Srivastava, V.; Mehta, D. Measurement of birefringence of nematic liquid crystal material by multiple-wavelength interferometry using nearly common-path single-stage Mach-Zehnder interferometer. Appl. Opt. 2013, 52, 8067-8072. [CrossRef]

41. Tang, S.; Kwok, H. Transmissive liquid crystal cell parameters measurement by spectroscopic ellipsometry. J. Appl. Phys. 2001, 89, 80-85. [CrossRef] 
42. Wu, S.; Efron, U.; Hess, L.D. Birefringence measurements of liquid crystals. Appl. Opt. 1984, 23, $3911-3915$. [CrossRef] [PubMed]

43. Özder, S.; Coskun, E.; Köysal, O.; Kocahan, Ö. Determination of birefringence dispersion in nematic liquid crystals by using an S-transform. Opt. Lett. 2007, 32, 2001-2003.

44. Nishiyama, I.; Yoshida, N.; Otani, Y.; Umeda, N. Single-shot birefringence measurement using radial polarizer fabricated by direct atomic force microscope stroking method. Meas. Sci. Technol. 2007, 18, 1673. [CrossRef]

45. Escuti, M.J.; Jones, W.M. A polarization-independent liquid crystal spatial light modulator. Proc. SPIE 2006, $6332,63320 \mathrm{M}$.

(C) 2020 by the authors. Licensee MDPI, Basel, Switzerland. This article is an open access article distributed under the terms and conditions of the Creative Commons Attribution (CC BY) license (http://creativecommons.org/licenses/by/4.0/). 